\title{
Optimal Scheme with Load Forecasting for Demand Side Management (DSM) in Residential Areas
}

\author{
Mohamed AboGaleela, Magdy El-Marsafawy, Mohamed El-Sobki \\ Electrical power and Machines department, Faculty of engineering Cairo University, Giza Egypt \\ Email: Eng.Abogaleela@gmail.com \\ Received February, 2013
}

\begin{abstract}
Utilities around the world have been considering Demand Side Management (DSM) in their strategic planning. The costs of constructing and operating a new capacity generation unit are increasing everyday as well as Transmission and distribution and land issues for new generation plants, which force the utilities to search for another alternatives without any additional constraints on customers comfort level or quality of delivered product. De can be defined as the selection, planning, and implementation of measures intended to have an influence on the demand or customer-side of the electric meter, either caused directly or stimulated indirectly by the utility. DSM programs are peak clipping, Valley filling, Load shifting, Load building, energy conservation and flexible load shape. The main Target of this paper is to show the relation between DSM and Load Forecasting. Moreover, it highlights on the effect of applying DSM on Forecasted demands and how this affects the planning strategies for utility companies. This target will be clearly illustrated through applying the developed algorithm in this paper on an existing residential compound in Cairo-Egypt.
\end{abstract}

Keywords: Component; Demand Side Management(DSM); Load factor(L.F.); Short Term Load Forecatsing(STLF); Long Term Load Forecasting(LTLF); Artificial Neural Network(ANN)

\section{Introduction}

The most common rationale for Demand Side Management in the Power Sector is that it is often more cost effective and socially beneficial to manage electricity demand through investment in efficiency and other demand side measures than to increase power supply or transmission capacity. DSM programs are used to eliminate or reduce the need for additional peak or base load generating capacity and/or distribution facilities. DSM also permits existing generation to meet the needs of a larger number of consumers or defers or reduces the need for new capacity.

Utilities, however, can benefit from these reductions or shifts in customer energy use. For some utilities, DSM programs can help them reduce their peak power purchases on the wholesale market, thereby lowering their overall cost of operations. In the short term, DSM programs can reduce energy costs for utilities, and in the long term, DSM programs can help limit the need for utilities to build new power plants, distribution, and transmission lines. In short, a DSM program can be much cheaper to implement than building a new generating plant.

\section{DSM Programs}

DSM Program is a strategy used to control the load pro- file indirectly in order to achieve the utility objectives. These objectives are [1]:

- To have the load factor as close as possible to 1.0.

- To have the peak load within the system capacity.

By achieving the previous objectives, the utility would get the maximum possible energy from the installed units, thus maximizing the total profit and minimizing the average cost per kWh.

Common techniques used for load shaping are peak clipping, valley filling, load shifting, strategic conservation, strategic load growth, and flexible load shape as shown in Figure 1 [2].

DSM program can also be classified into [3]:

- EE (Energy efficiency) programs which are designed to reduce electricity consumption throughout the year by focusing on reducing energy consumption and overall energy demand.

- DR (Demand Response) programs which are automatic with a processing unit having the right to moderate or turn-off certain appliances (e.g. air-conditioners, pool pumps, washing machines, etc.) for a short period of time at customer sites.

\section{Load Forecasting}

Electric load forecasting is an important aspect in elec- 
trical power industry. It is important to determine the future demand for power as far in advance as possible. According to the foreseen load the company makes investments and decisions on buying energy from the generating companies, and planning for maintenance and expansion. It is therefore absolutely necessary to have some knowledge of future power consumption. Electric power distributors require a tool that allows them to predict the load in order to support its management and make more efficient in planning formulation. Accurate prediction of electric load is difficult. A large number of the classical prediction models are inappropriate for this modeling because of their requirement [4].

The prediction of the electric load at a future time is a challenging problem because of the diverse characteristics of the electrical load and the uncertainly associated with them. A typical daily variation of electric loads is shown in Figure 2. The characteristics of the electric load depend on the nature of the users and the end use devices utilized such as motors, air conditioners, lighting, etc. From this point of view the electric load can be separated into four major categories [5].

- Residential

- Commercial

- Agricultural

- Industrial
In a few words load forecasting is very important now days for the utility companies especially those using the DSM alternatives, as DSM alternatives will be applied on the forecasted load demand instead of the existed load.

Importance of load forecasting in the deregulated market is considered as follows:

- Purchasing, generation, sales

- Contracts

- DSM

- Area planning

- Infrastructure development/capital expenditure decision making

Load Forecasting is divided into three main Types:

- short-term load forecasting (STLF)

- medium-term load forecasting (MTLF)

- long-term load forecasting (LTLF)

Load Forecasting Methods

Over the last few decades a number of forecasting methods have been developed. The different forecasting methods could be stated as follows [6]:

- Regression Models.

- Time serried approaches.

- Similar day approach.

- Artificial Neural Networks (ANN).

ANN is used as the forecasting tool presented in this paper.
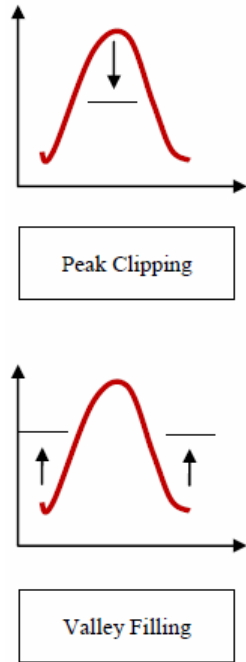

Figure 1. Load shape objectives.
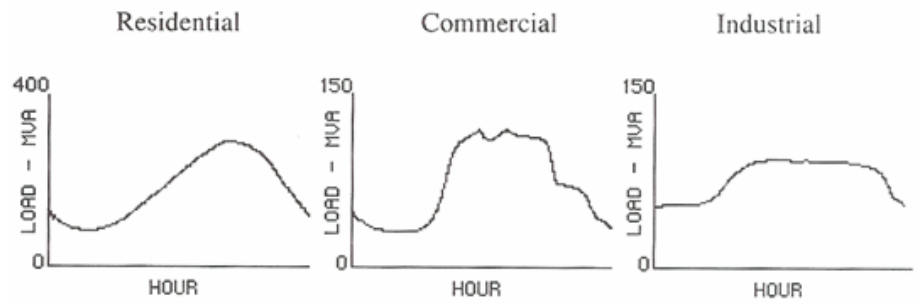

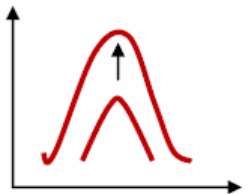

Load Building

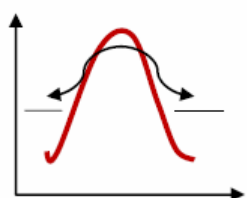

Load Shifting

Figure 2. Typical daily load variation. 


\section{Simulation Tools}

In this paper three tools are introduced:

\subsection{Load Forecasting Tool (ANN)}

The use of artificial neural networks (ANN or simply NN) has been a widely studied electric load forecasting technique since1990 [7] Neural networks are essentially non-linear circuits that have the demonstrated capability to do non-linear curve fitting. The outputs of an artificial neural network are some linear or nonlinear mathematical function of its inputs. The inputs may be the outputs of other network elements as well as actual network inputs. In practice network elements are arranged in a rela- tively small number of connected layers of elements between network inputs and outputs. Feedback paths are sometimes used.

ANN used is MATLAB based.

\subsection{Smart Meter Tool}

A smart tool named-SEP2 Manager/ISKRA- is used through this work having the following features:

- Hour by Hour power consumption is recorded through the meter.

- Generates the data to Excel sheets.

- Plotting the overall daily load curve for the whole compound.

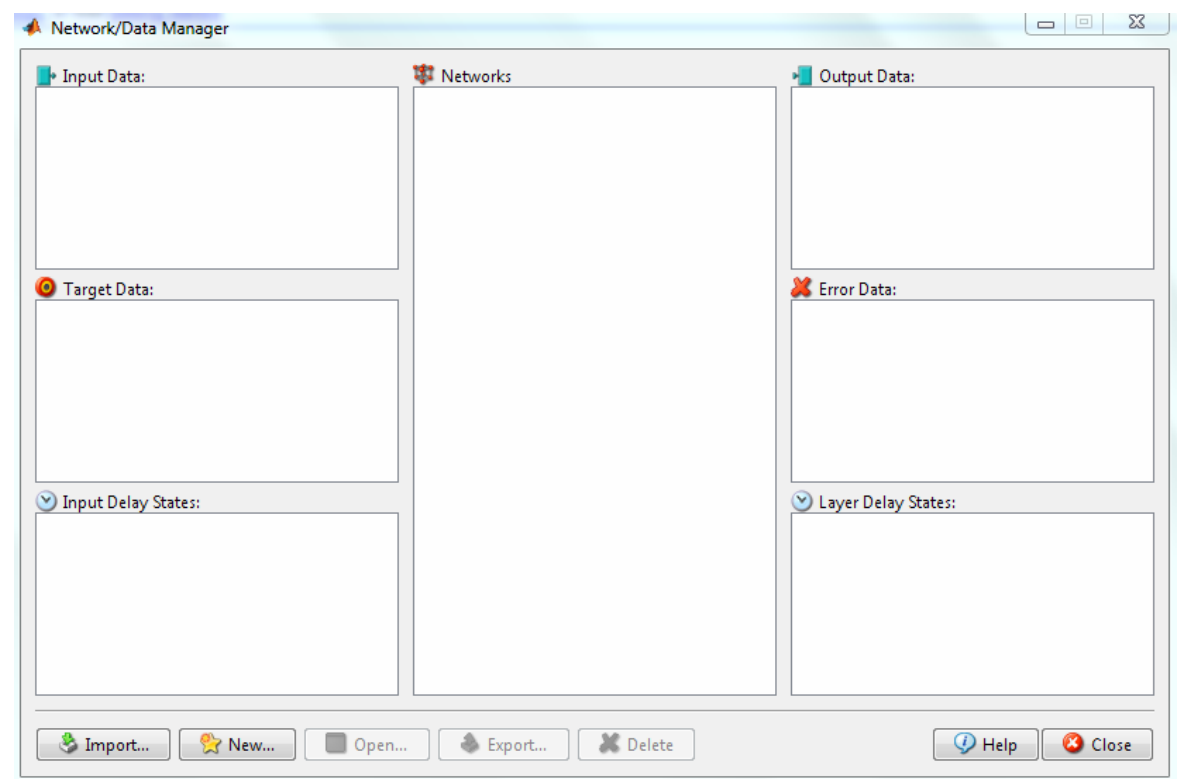

Figure 3. MATLAB ANN tool main page.

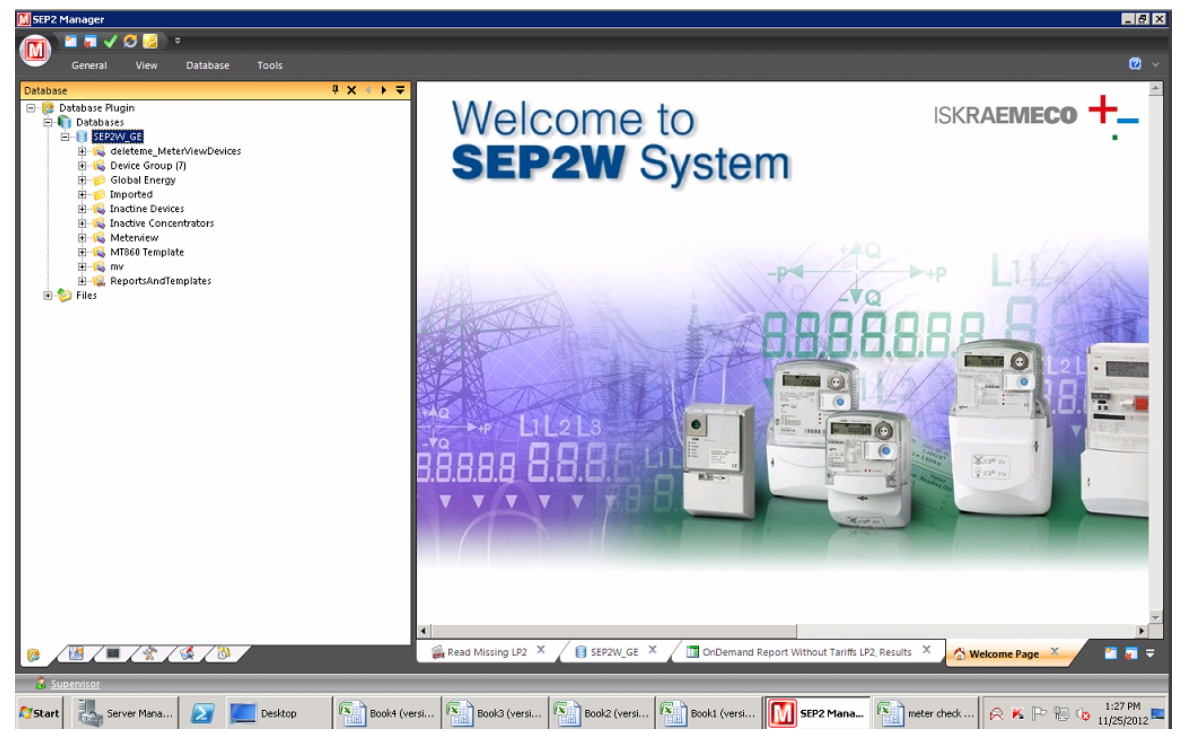

Figure 4. Main page of Smart meter tool[9]. 


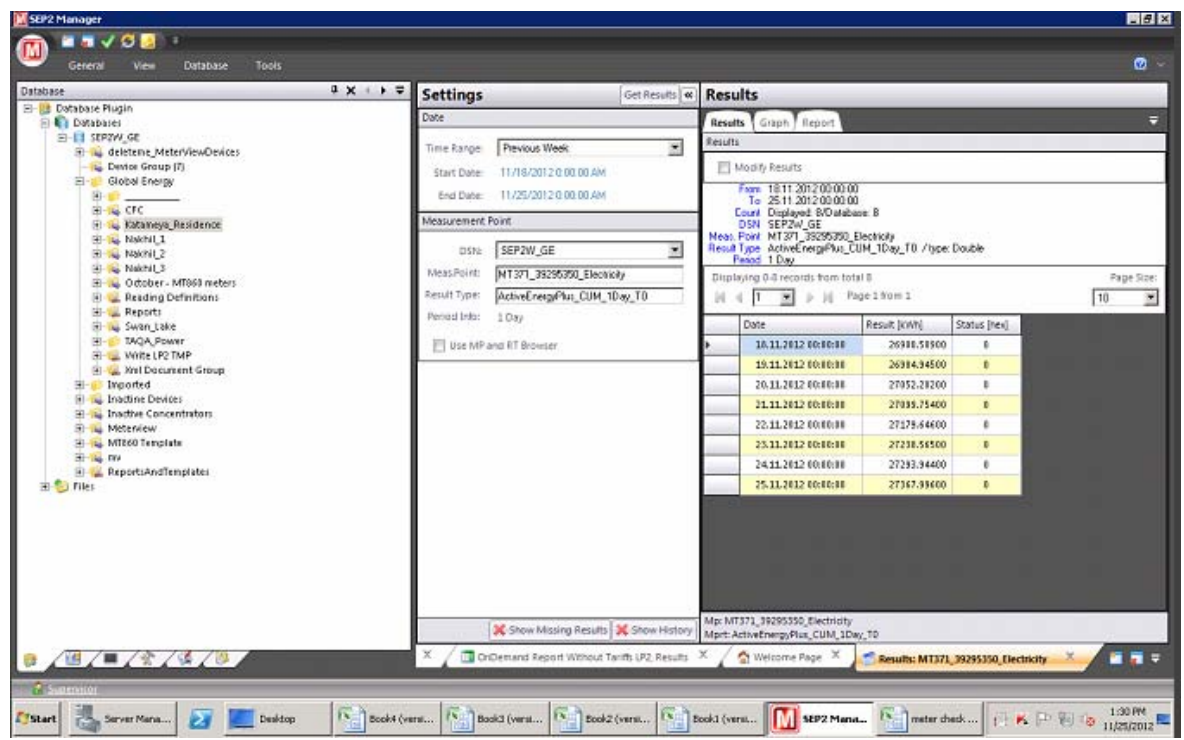

Figure 5. Generated Data tables screen[9].

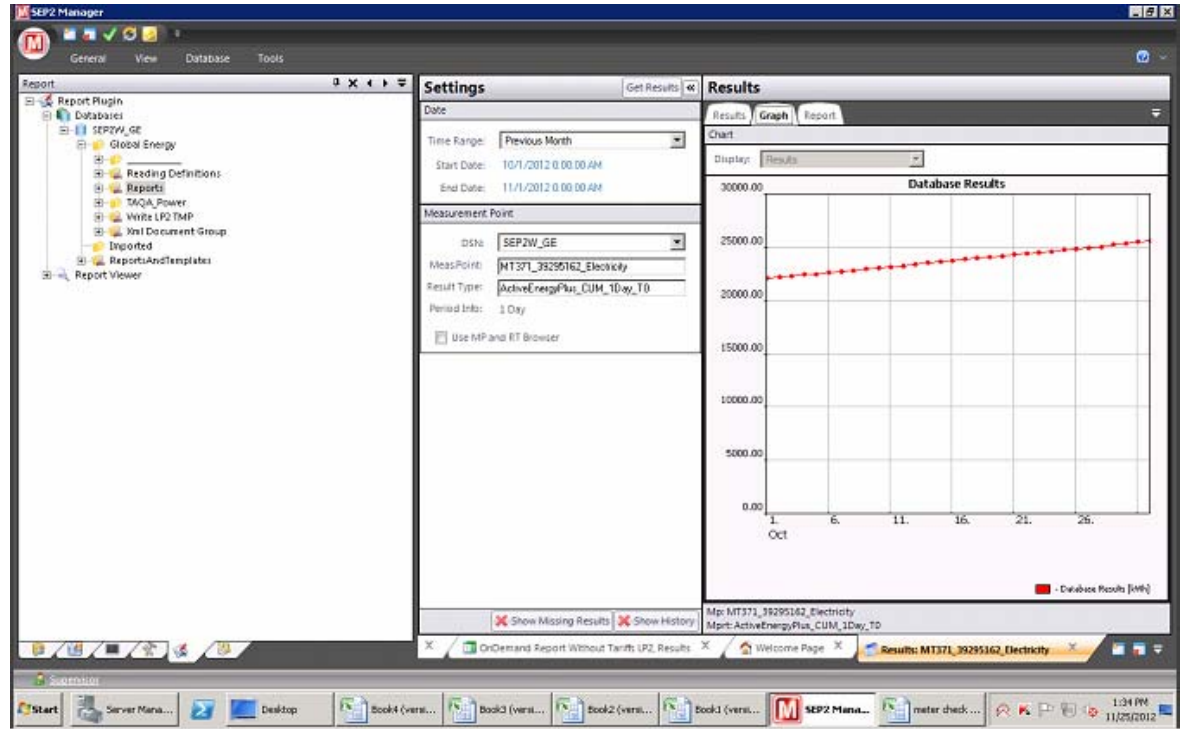

Figure 6. Output load curves from the tool[9].

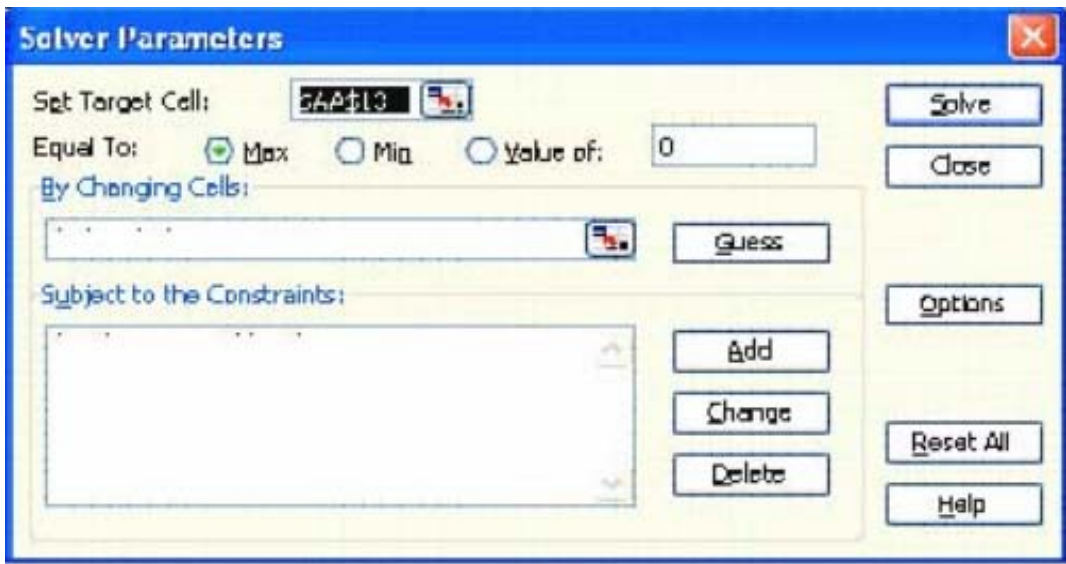

Figure 7. Solver Main Screen. 


\subsection{DSM Optimization Tool (Solver-Excel)}

The DSM solution tool used is SOLVER EXCEL based used for optimization. The main screen of solver is shown in figure 6. The objective function is written in the target cells and the constraints are written in the changing cells.

\section{Practical Case Study with Algorithm Description}

Our case is Real estate development located in New Cairo, Egypt which is fed by electric power on the $22 \mathrm{kV}$ with contracted capacity till 2012 is $20 \mathrm{MW}$. This compound is fed through a private sector company.

In this section load forecasting is applied using ANN on the previous case study. Where the day of the maximum demand for the previous compound was in the first week of August, so forecasting is applied to estimate the demand of this day in the next previous three years (2013, 2014\&2014).Then DSM load shifting is applied on the forecasted demand.

The Algorithm steps could be summarized as follows:

- The Historical data of the load is obtained from the tool.

- The historical data for the weather is obtained from the weather forecasting Agencies.

- These data are inserted to the ANN toolbox based under MATLAB.

- A forecasting model is developed based on the data entry to the ANN toolbox.

- A validation step should be made to the developed forecasting model.

- The model generates the new load demand at certain required days through entering the weather conditions at these years..

- Finally the new loads curves are plotted through excel.

- DSM is Load shifting is applied on the new forecasted load curves.

\section{Problem Formulation for DSM}

A DSM optimization algorithm is introduced in order to shift loads for achieving the following objective function which is maximizing load factor of the system with reducing residual Area between load and supply (i.e. increasing shared area between supply and load) and this DSM will be applied in the forecasted load curves that its load curve exceeds the supply curve in some regions [8].

Objective function:

Max L.F. $=\left[\left[\frac{\left[\sum^{\mathbf{i}=1 \mathbf{i}=\mathbf{N}} \sum^{\mathbf{j}=1 \mathbf{j}=\mathbf{J}} \mathbf{p}(\mathbf{i}, \mathbf{j}) * \mathbf{t}(\mathbf{j})\right]}{\sum^{\mathbf{j}=\mathbf{j}=\mathbf{J}} \mathbf{t}(\mathbf{j})}\right] / \mathbf{P T O}(\mathbf{k})\right]$

where:
- L.F.: is the system load factor.

- $\mathrm{P}(\mathrm{i}, \mathrm{j}))$ : is the demand of load type $\mathrm{i}$ at time interval number $\mathrm{j}$.

- $\mathrm{N}$ : is the total number of load demand types.

- $\mathrm{J}$ : is the total number of time intervals.

- PTO (j): is the total demand for all the loads types from $j=1$ to $j=J$ over the time interval number $j$.

- $\mathrm{K}$ : is the number of time interval at which the maximum demand for all the load types numbers from $\mathrm{i}=1, \mathrm{~N}$ over all the time duration from $\mathrm{j}=1, \mathrm{~J}$ occurs.

- PTO (K): is the maximum of total demand for all the loads types.

The previous objective function is subjected to the following constraints:

- Equality constraint: Energy consumption is the same before and after shifting.

$$
\sum_{\mathbf{i}=1}^{\mathrm{i}=\mathbf{N} \mathbf{j}=\mathbf{J}=1} \operatorname{pnew}(\mathbf{i}, \mathbf{j}) * \mathbf{t}(\mathbf{j})=\sum_{\mathbf{i}=1}^{\mathrm{i}=\mathbf{N} \mathbf{j}=\mathbf{J}} \mathbf{j}=1
$$

- Inequality constraint: Difference between supply and load should be positive.

$$
\mathbf{s}(\mathbf{j})-\mathbf{P T O}(\mathbf{k})>\text { zero }
$$

- Inequality constraint: Peak demand after applying shifting algorithm should be less than that before shifting.

$$
\text { pmax_new }<\text { pmax_old }
$$

where:

- Pnew $(i, j)$ is the new power of each load type(i) at time interval(j) after applying shifting algorithm.

- Pold $(\mathrm{i}, \mathrm{j})$ is the old power of each load type(i) at time interval(j) before applying shifting algorithm.

- PTO (j): is the total load power at time interval (j).

- $S(\mathrm{j})$ : is the supply value at time interval $\mathrm{j}$.

\section{Simulation Results}

\subsection{Load Forecasting Results}

Figure 9 explains the validation stage for the forecasting model through comparing the output demand profiles from the model at certain days where the actual demand profiles are known at those days.

Figure 10 indicates the mean square error that measures the deviation between the actual and forecasted demands.

Figure 11 shows the curve fitting procedure that is made through the ANN tool box during the forecasting stage.

From the previous tables and curves the following comments were noticed:

- The load curve at peak day of year 2013 is maintained within the supply.

- The load curves at peak days of years 2014 and 
2015 are exceeding the supply capacity at certain times.

- The utility should put in the capacity expansion plan starts from now-2012- so they could meet the demand requirements by the years 2014 and 2015 .

- The utility could apply one of the DSM alternatives on the forecasted demand to achieve the best match between the load and supply and this will be illustrated in the following section.

\subsection{DSM Load Shifting Results}

DSM load shifting is applied in the load curves of years 2014 and 2015 as the load curve exceeds the supply curve at the peak day in these years and the resultant curves after applying DSM are as follows:

From the previous figures the following comments are deduced as follows:

- The role of applying DSM LS algorithm in matching the load with the supply.

- The peak demand is reduced.

- The utility will postpone its investments to meet the load requirements.

- The Load Factor of the system is increased.

- The importance of applying of integrating DSM and load forecasting in the planning studies for utility companies.

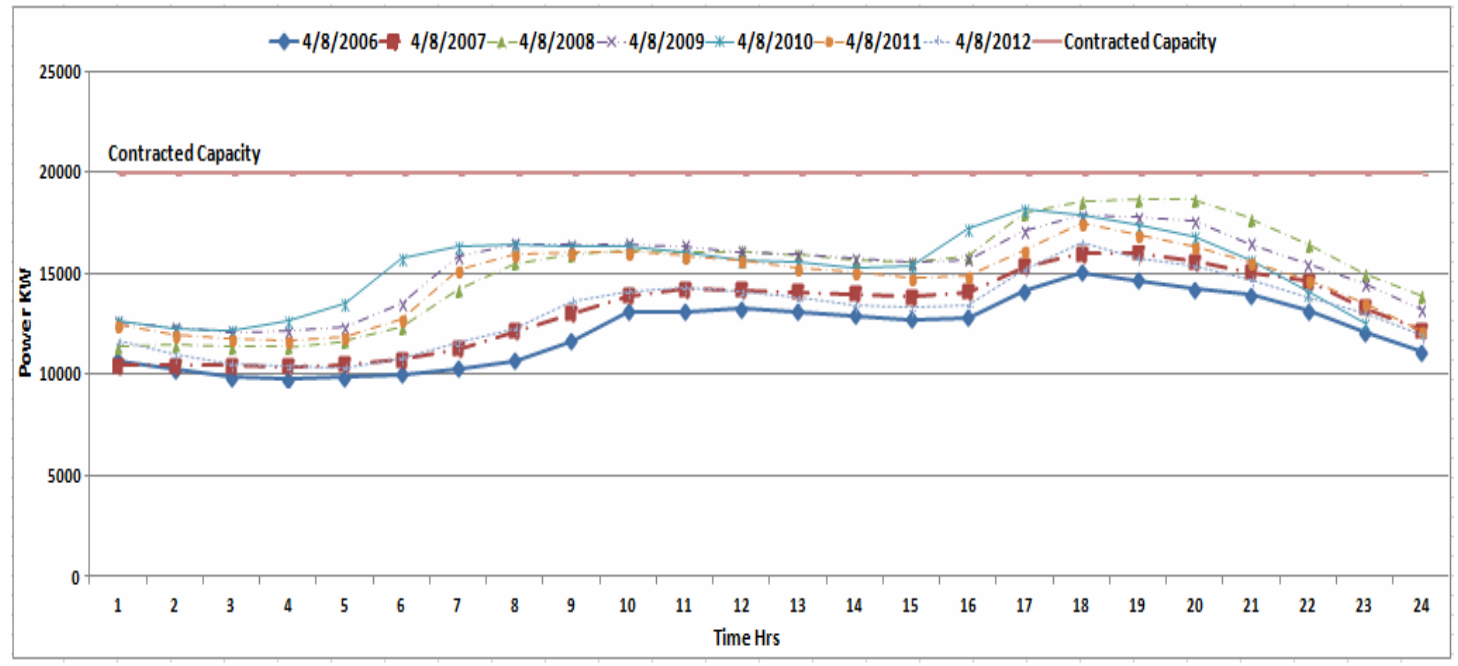

Figure 8. Historical Load curves.

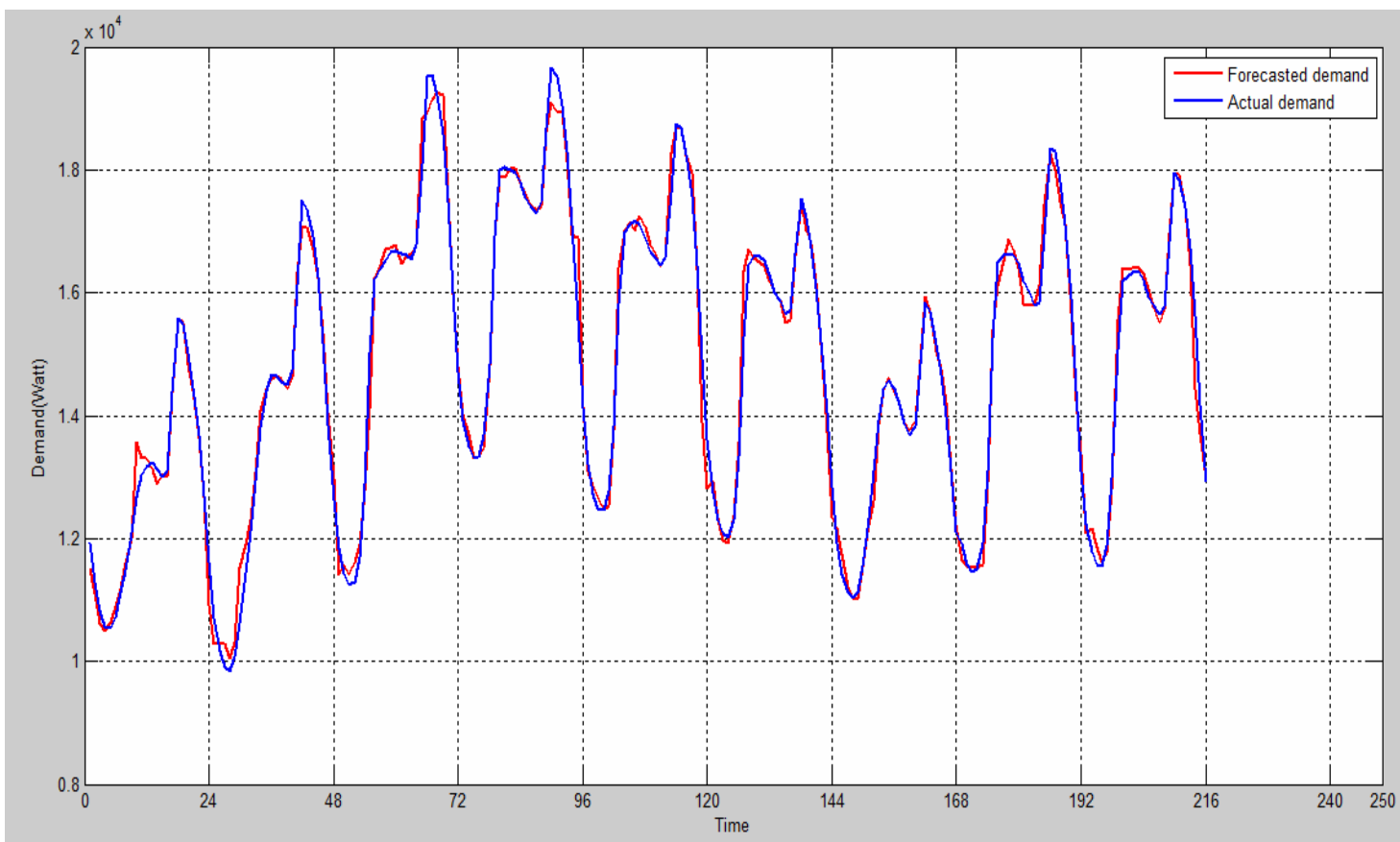

Figure 9. Forecasting model validation. 


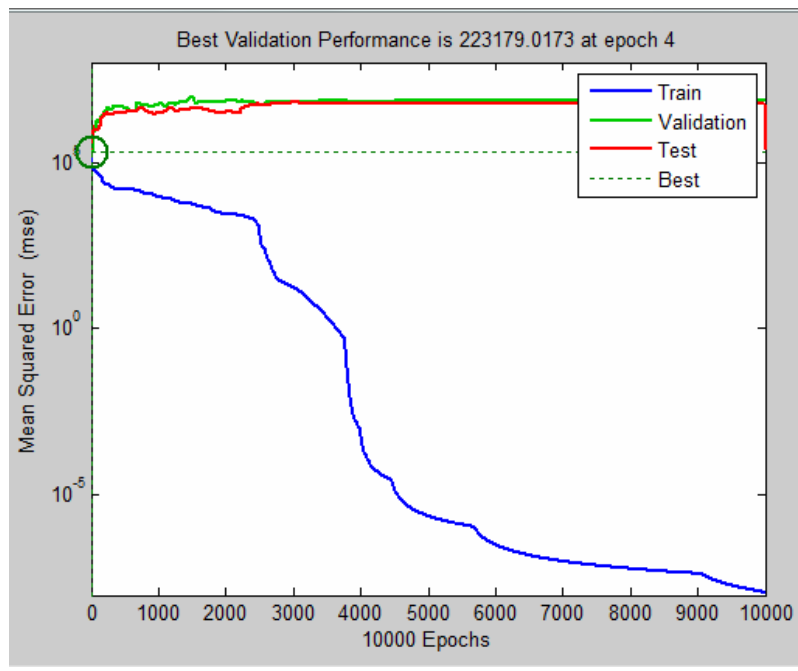

Figure 10. ANN behavior Mean square error.

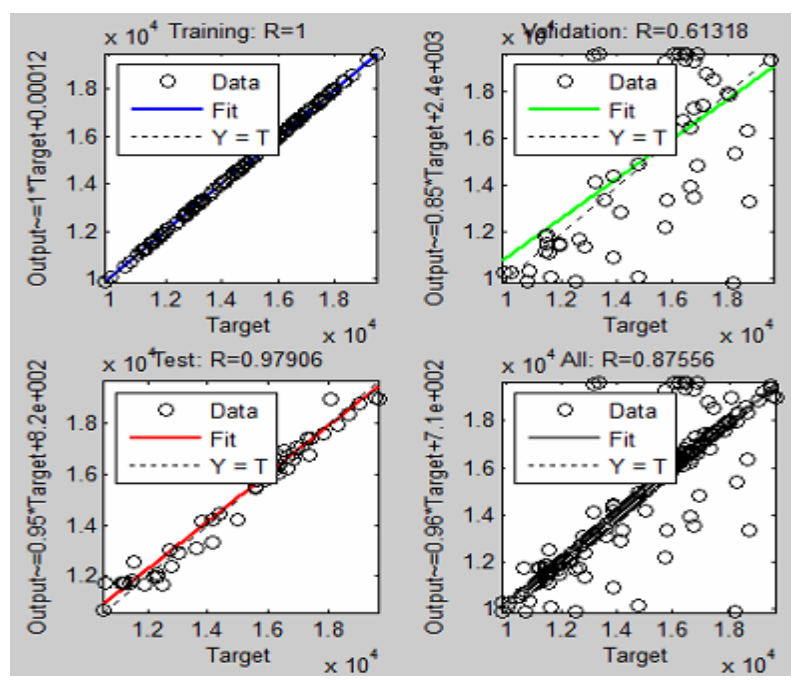

Figure 11. Regression Curves from ANN tool box.

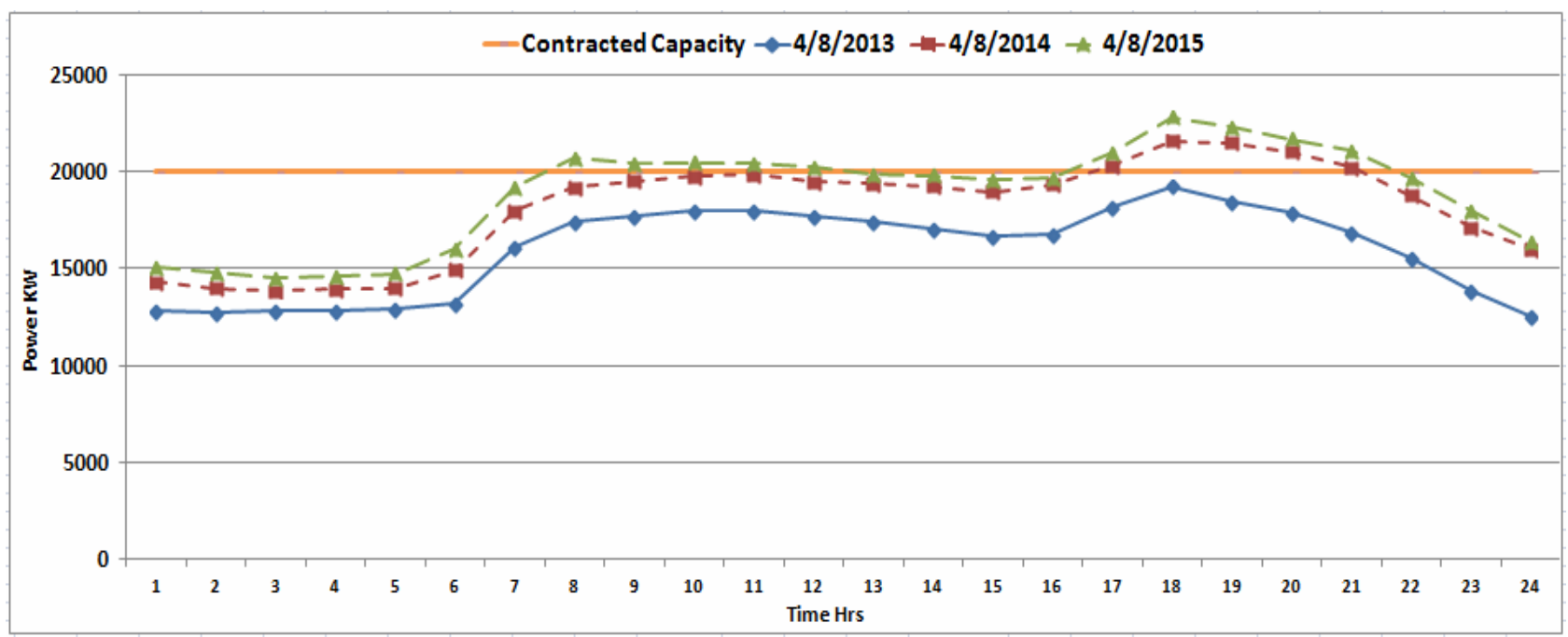

Figure 12. Peak day forecast at 2013, 2014 and 2015.

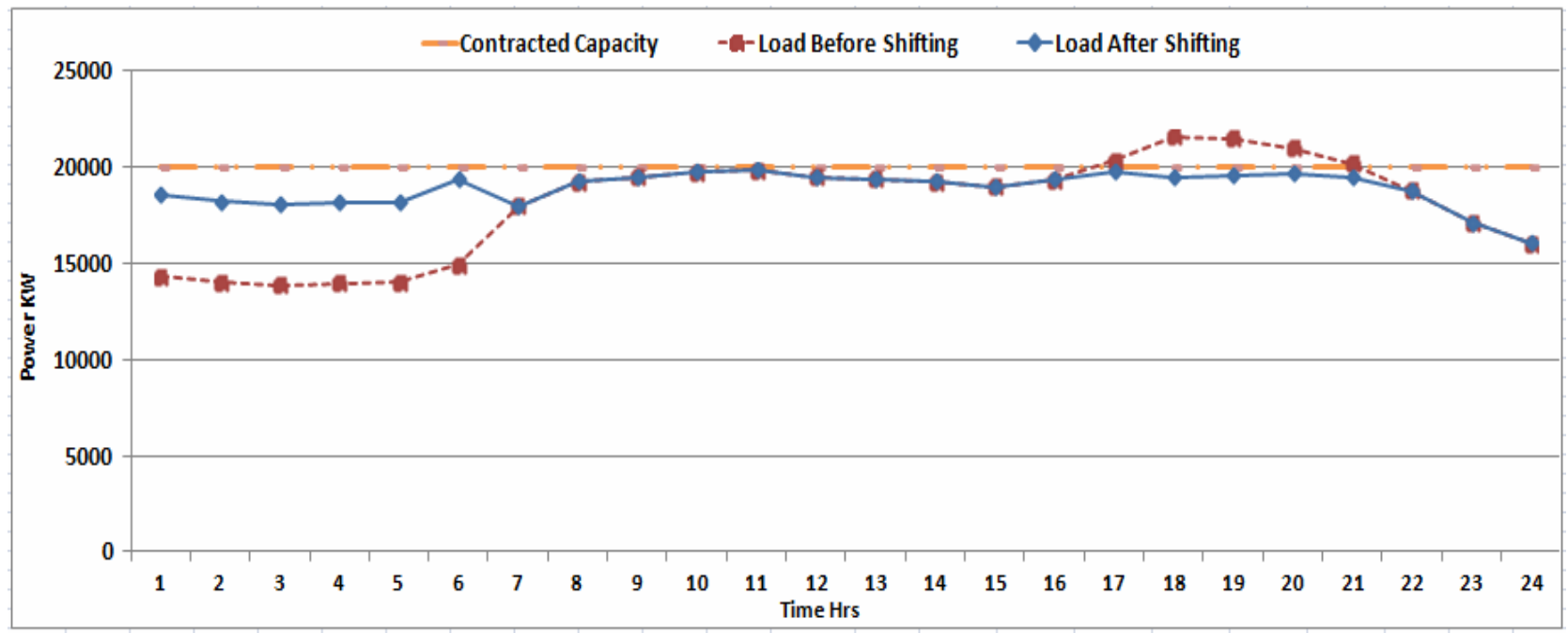

Figure 13. Load curves before and after shifting vs. day time in 2014. 


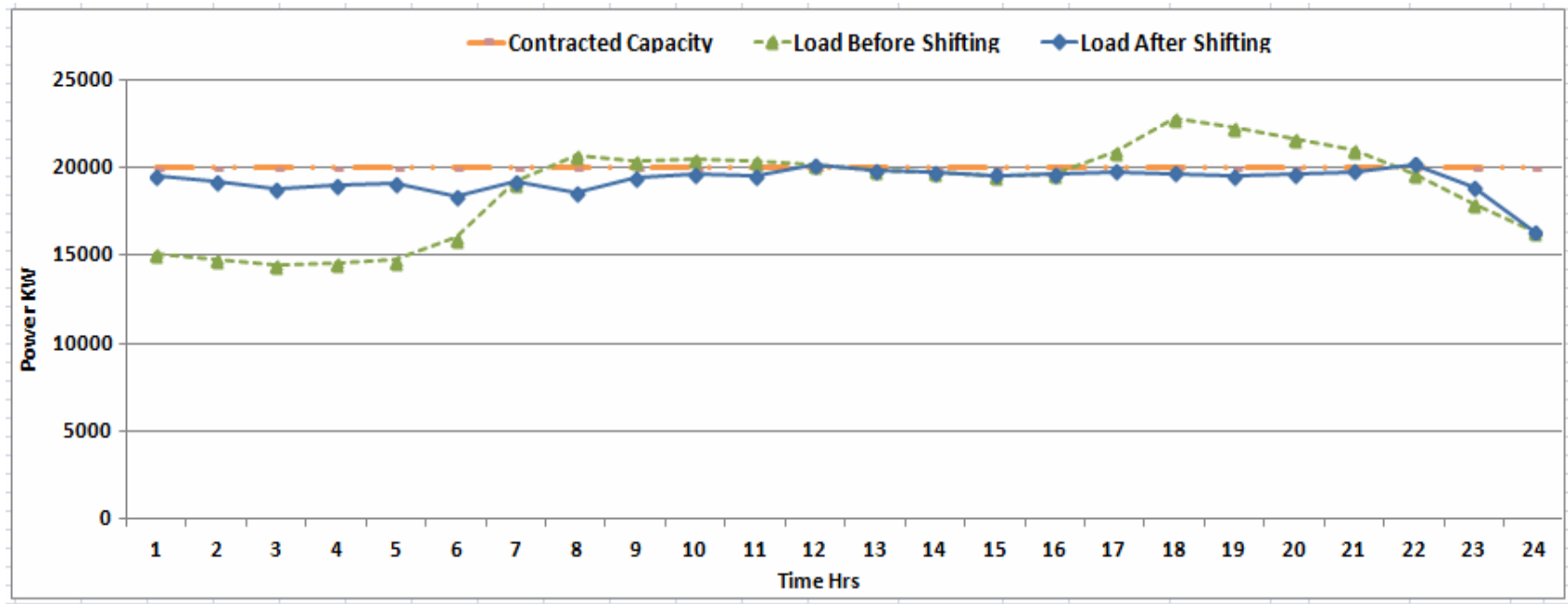

Figure 14. Load curves before and after shifting vs. day time in 2015.

\section{Conclusions}

In this paper a integrated scheme of DSM with load forecasting is introduced over a practical case where the following comments were noticed:

- Impact of applying load forecasting and knowing the peak demand.

- The impact of DSM in matching load curve with the supply curve.

- The impact of DSM in reducing the peak demand of the system.

- The importance of applying of integrating DSM and load forecasting in the planning studies for utility companies.

- The utility will postpone its investments in expanding the generation capacities to meet the load.

\section{REFERENCES}

[1] H. Attia, "Mathematical Formulation of the Demand Side Management (DSM) Problem and its Optimal Solution," Proceedings of the 14th International Middle East Power Systems Conference (MEPCON'10), Cairo University, Egypt, December 19-21, 2010.

[2] Electric Power Research Institute, -Demand-side Management: Utility Options for the Future, \| EPRI Reports,
CU. 3028.10.89, 2006.

[3] N. Kanoksing and W. Tayati, "Economics of Demand Side Management and Hybrid Renewable Energy System for a Remote Village Electrification in Northern Thailand, AUPEC'07, Curtin University of Technology, Perth, Australia, Dec 2007.

[4] R. Achnata, "Long Term Electric Load Forecasting using Neural Networks and Support Vector Machines," IJCST, Vol. 3, No. 1, 2012.

[5] R. Holmukhe, "Artificial Neural Network based short term load forecasting technique for Indian power system energy management," College of Engineering Bhanti dyapeetb Diversity, Pune43, 2007.

[6] E. Feinberg, Applied Math \& Statistics Stony Brook University NSF workshop, November 3-4, 2003.

[7] M. Peng, N. F. Hubele and G. G. Karady, "Advancement in the Application of Neural Networks for Short-Term Load Forecasting," IEEE Transactions on Power Systems, Vol. 7, 1992, pp. 250-257. doi:10.1109/59.141711

[8] M. Abogaleela, M. Elsobki and M. ElMarsafawy, "A Two Level Optimal DSM Load Shifting Formulation Using Genetics Algorithm, Case study: Residential load, Power Africa 2012.IEEE PES, South Africa.

[9] Smart Meter "Iskra" Tool Owned by "TAQA Power" Company. 